\title{
Cerâmicas odontológicas na confecção de facetas laminadas: qual a melhor escolha?
}

\author{
Lairds Rodrigues dos Santos*, Claudia Maria Coelho Alves \\ Programa de Pós-Graduação em Odontologia da Universidade Federal do Maranhão - UFMA, São Luís, MA, Brasil.
}

Histórico do Artigo:

Recebido em:

28/09/2020

Aceito em:

$26 / 10 / 2020$

Palavras-chave:

Cerâmica; facetas

dentárias; cimentação

Keywords:

Ceramics; dental

veneers; cementation

\begin{abstract}
RESUMO
A demanda por restaurações estéticas tem resultado em um aumento do uso das cerâmicas dentais, constituindo a principal alternativa de material restaurador. Além de proporcionarem um grande mimetismo das estruturas dentárias elas promovem a restauração da camada dentária perdida, devolvendo a resistência mecânica ao dente e garantindo a estabilidade de cor. O propósito desta revisão foi mostrar as possibilidades da reabilitação estética com cerâmicas odontológicas para tratamento restaurador com facetas laminadas. Artigos científicos, disponíveis nas bases de dados Pubmed, Google acadêmico, Scielo, portal Capes e em revistas relacionadas ao assunto foram consultados. Também foram pesquisados livros e websites. Verificou-se na literatura pesquisada que o principal material de escolha para a confecção de facetas laminadas é o dissilicato de lítio, pois quando comparada aos outros, eles apresentam uma melhor capacidade adesiva, aperfeiçoamento no processo de confecção laboratorial, resistência flexural triplicada, além de excelente estética, translucidez, opacidade e fluorescência semelhantes ao do dente. As porcelanas à base de dissilicato de lítio são as mais indicadas para a confecção de facetas laminadas por apresentarem maior resistência flexural, excelente estética e melhor capacidade adesiva em comparação a outras cerâmicas odontológicas.
\end{abstract}

Dental ceramics in the making of laminated facets: what is the best choice?

\section{ABSTRACT}

The demand for a esthetic restorations has resulted in an increased use of dental ceramics, constituting the main alternative for restorative material. In addition to offering great mimicry of dental structures, they promote the restoration of the lost tooth layer, restoring mechanical strength to the tooth and preventing color stability. The purpose of this review was search the literature as possibilities of aesthetic rehabilitation with dental ceramics for restorative treatment with laminated facets. Scientific articles, available in the databases Pubmed, Google Scholar, Scielo, Capes portal and in magazines related to the subject were consulted. Books and websites were also searched. It was found in the researched literature that the main material of choice for making laminated veneers is lithium disilicate, because when compared to the others, they have a better adhesive capacity, improvement in the laboratory making process, triple flexural strength, in addition to excellent esthetics, translucency, opacity and fluorescence similar to that of the tooth. Lithium disilicate porcelains are the most suitable for making laminated veneers because they have greater flexural strength, excellent aesthetics and better adhesive capacity compared to other dental ceramics.

\section{Introdução}

Dentre os materiais restauradores estéticos, a cerâmica pode ser considerada a melhor escolha para reproduzir os dentes naturais, devido às suas propriedades físicas, biológicas e ópticas, que permitem a manutenção da cor com o passar do tempo. Além disso, apresentam resistência à abrasão, grande estabilidade no meio bucal e biocompatibilidade (1).

A estética é um fator primordial na Odontologia. As restaurações cerâmicas, por sua vez, em razão de sua alta capacidade de mimetizar os tecidos dentais, ainda são a

\footnotetext{
*Autor correspondente: lairds_odonto@yahoo.com.br (dos Santos L.R.)
} 
escolha de muitos profissionais e pacientes (2). Entretanto, as tradicionais coroas metalocerâmicas consistem numa infraestrutura metálica recoberta por porcelana, que por ser opaca não consegue imitar a translucidez do dente natural ${ }^{3}$. Assim, os sistemas totalmente cerâmicos (livres de metal) surgiram com intuito de eliminar esta infraestrutura, na tentativa de promover uma melhor distribuição da reflexão da luz, resultando em uma melhor estética $(2,3)$.

Contudo, vale ressaltar que, os sistemas totalmente cerâmicos são frágeis e de baixa resistência mecânica, quando submetidas às tensões de tração, o que compromete seu desempenho clínico em alguns aspectos (3). Ocasionalmente, estas cerâmicas falham gerando desconforto e inconvenientemente abrem questões sobre a efetividade desse tipo de tratamento ${ }^{2}$. Dessa forma, as cerâmicas têm apresentado rápida evolução em âmbito científico, a fim de melhorar suas propriedades físicas e mecânicas, para suprir as necessidades estéticas exigidas pela sociedade moderna $(2,4)$.

Por conta disso, definir a efetividade e determinar a média de longevidade tem sido o principal objetivo dos estudos com cerâmicas odontológicas, especialmente no que se refere às qualidades de adesão, biocompatibilidade, resistência ao manchamento e corrosão, bem como, características ópticas similares às das estruturas dentárias $(2,4)$.

A faceta laminada é um procedimento estético totalmente cerâmico realizado com o recobrimento da face vestibular da estrutura dental, fortemente unido ao elemento dentário por meio de sistemas adesivos $(5,6)$. Entretanto, critérios concretos a respeito da durabilidade e longevidade de procedimentos com facetas laminadas, são difíceis de serem conseguidos, uma vez que, o sucesso depende de alguns fatores, dentre eles: tipo de material, técnica adesiva e tipo do agente cimentante utilizados (7).

Assim, novos materiais e técnicas para confecção dessas restaurações totalmente cerâmicas foram introduzidos no mercado ampliando a diversidade de tipos de cerâmicas odontológicas existentes, melhorando não só nas propriedades estéticas, como também, físicas e mecânicas $(4,8)$.

Considerando os aspectos abordados, o propósito da presente revisão de literatura é apresentar as cerâmicas odontológicas mais utilizadas na confecção de facetas laminadas, enfatizando tipo e composição, vantagens, limitações, bem como o tipo de tratamento de superfície das cerâmicas, tendo em vista que a necessidade de conhecimento destes materiais e seus protocolos de preparo da superfície dentária são importantes para o sucesso clínico com longevidade, estética e correta reabilitação protética.

\section{Material e Métodos}

Para esta revisão, foram utilizadas publicações disponíveis em meio eletrônico ou impresso. Artigos científicos nas bases de dados Pubmed, Google acadêmico, Scielo, Lilacs e o portal Capes, entre outras foram utilizadas. Também foram consultados livros e websites. O período de busca foi de janeiro a setembro de 2020. Foram utilizados como descritores para a busca: cerâmica, facetas dentárias e cimentação, e seus termos na língua inglesa respectivamente, ceramics, dental veneers e cementation, segundo o Medical Subject Headings (MeSH)/PubMed. Somente estudos na língua inglesa e portuguesa que se referiam a utilização das cerâmicas na aplicação odontológica foram incluídos nesta revisão. Os artigos selecionados compreenderam o ano de 2004 a 2020. Foram excluídos artigos repetidos nas bases de dados, artigos que não estavam na íntegra, ensaios não controlados, e os que não apresentavam qualquer relação com a seguinte questão norteadora: Cerâmicas odontológicas na confecção de facetas laminadas. Qual a melhor escolha? Sendo selecionados os textos científicos que 
apresentavam na íntegra o real objetivo do trabalho.

\section{Revisão de Literatura}

\section{Facetas laminadas}

As cerâmicas são utilizadas com frequência na clínica odontológica, seja para solucionar a perda de um único dente ou na reabilitação de toda oclusão. As restaurações cerâmicas livres de metal têm se tornado a principal alternativa de tratamento para a reconstrução das estruturas dentárias (3).

Dessa forma, as facetas laminadas surgem como uma alternativa restauradora estética que alia a possibilidade de minimizar o desgaste dental requerido durante a fase de preparo e a de proporcionar uma modificação estética significativa e duradoura (9). Confeccionada pela técnica indireta, este tratamento proporciona propriedades ópticas, mecânicas e biológicas semelhantes às do esmalte natural $(5,6)$.

Os laminados cerâmicos ultrafinos conhecidos como "lentes de contato dentais" consistem em restaurações com espessuras de 0,2 a $0,5 \mathrm{~mm}$, que são cimentadas sobre a face vestibular dos dentes e demandam um mínimo ou nenhum desgaste do esmalte dental, indicadas para corrigir pequenas alterações de cor, forma e tamanho, se estabelecendo como uma modalidade efetiva de tratamento (10,11). Já as facetas laminadas convencionais também são um tipo de laminado cerâmico, sendo um pouco mais espessas que as lentes de contato, entre 0,6 a 1,2 $\mathrm{mm}$. Tendo como indicações os problemas dentais quanto à forma, posição, simetria, textura superficial e cor $(12,13)$.

Com a melhoria dos materiais e das técnicas de fabricação, diferentes alternativas cerâmicas são oferecidas para os dentistas com o intuito de aumentar as taxas de sucesso para esse tipo de tratamento (14). Porém, algumas etapas devem ser seguidas durante o processo de reabilitação protética com cerâmicas: planejamento adequado do caso, preparo conservador, preservando o máximo de esmalte, eleição adequada de cerâmicas para uso, seleção apropriada dos materiais e métodos de cimentação $(15,16)$.

Assim, tendo em vista que a excelência estética é abordada como referência nos tratamentos de facetas laminadas, para se obter sucesso com esse tratamento, o conhecimento dos tipos de cerâmicas, torna-se uma prioridade e a habilidade na técnica adesiva torna-se fundamental (17).

\section{Considerações gerais das cerâmicas}

Existe uma grande variedade de classes cerâmicas disponíveis para distintas indicações, de acordo com seus fabricantes (2). Segundo Gomes et al. (18) as cerâmicas odontológicas são compostas por elementos metálicos (alumínio, cálcio, lítio, magnésio, potássio, sódio, lantânio, estanho, titânio e zircônio) e substâncias não metálicas (silício, boro, flúor e oxigênio).

Estas cerâmicas podem ser divididas quanto ao conteúdo (composição) em: vítreas e cristalinas/policristalinas. A fase cristalina é circundada por uma fase vítrea de silicato (19). Ainda, quanto ao tipo, são classificadas em: cerâmicas convencionais (feldspáticas) e cerâmicas reforçadas (leucita ou dissilicato de lítio) infiltradas (alumina, alumina e magnésio, alumina e zircônia) e policristalinas $(19,20)$. A seguir tem-se o Quadro 1, que mostra o comparativo dos tipos e características gerais das cerâmicas odontológicas. 
Quadro 1 - Características das cerâmicas feldspáticas, reforçadas, infiltradas e policristalinas

\begin{tabular}{|c|c|c|c|c|c|}
\hline Cerâmica & Fabricante & Indicações & $\begin{array}{l}\text { Característica } \\
\mathrm{s}\end{array}$ & $\begin{array}{l}\text { Tratamento } \\
\text { de superfície }\end{array}$ & $\begin{array}{c}\text { Resistênci } \\
\text { a flexural } \\
(\mathrm{MPa})\end{array}$ \\
\hline Feldspáticas & $\begin{array}{c}\text { Duceram Plus, } \\
\text { Duceram Kiss } \\
\text { (Degudent); Super- } \\
\text { Porcelain EX3, } \\
\text { Cerabien (Noritake) }\end{array}$ & $\begin{array}{c}\text { Cobertura de } \\
\text { infra-estrutura } \\
\text { metálica } \\
\text { (metalocerâmica } \\
\text { s) Inlays, onlays } \\
\text { e facetas } \\
\text { laminadas } \\
\text { Recobrimento } \\
\text { de infraestrutura } \\
\text { cerâmica (livre } \\
\text { de metal) } \\
\end{array}$ & $\begin{array}{l}\text { Matriz vítrea } \\
\text { com partículas } \\
\text { cristalinas } \\
\text { dispersas }\end{array}$ & $\begin{array}{c}\text { Ácido } \\
\text { sensíveis }\end{array}$ & $20-60$ \\
\hline $\begin{array}{c}\text { Reforçadas com } \\
\text { leucita }\end{array}$ & $\begin{array}{c}\text { IPS Empress (Ivoclar } \\
\text { Vivadent), Optimal } \\
\text { Pressable Ceramic } \\
\text { (Jeneric Pentron), IPS } \\
\text { ProCAD (Ivoclar } \\
\text { Vivadent), IPS } \\
\text { Empress Esthetic } \\
\text { (Ivoclar Vivadent) } \\
\end{array}$ & $\begin{array}{c}\text { Inlays, onlays e } \\
\text { facetas } \\
\text { laminadas } \\
\text { Coroas } \\
\text { anteriores } \\
\text { Recobrimento } \\
\text { de infraestrutura } \\
\text { cerâmica } \\
\end{array}$ & $\begin{array}{l}\text { Matriz vítrea } \\
\text { com reforço de } \\
\text { partículas de } \\
\text { leucita }\end{array}$ & $\begin{array}{c}\text { Ácido } \\
\text { sensíveis }\end{array}$ & $97-180$ \\
\hline $\begin{array}{l}\text { Reforçadas com } \\
\text { dissilicato de } \\
\text { lítio }\end{array}$ & $\begin{array}{c}\text { IPS Empress } ® 2, \text { IPS } \\
\text { e.max® Press } \\
\text { (Injetado), IPS e.max® } \\
\text { CAD (fresado), } \\
\text { (Ivoclar Vivadent); } \\
3 \text { G }^{\mathrm{TM}} \text { OPC (Pentron } \\
\text { Ceramic Inc.), } \\
\text { Obsidian (Glidewell } \\
\text { Laboratories), } \\
\text { Suprinity (Vita), Celtra } \\
\text { Duo (Dentsply) }\end{array}$ & $\begin{array}{l}\text { Inlays, onlays, } \\
\text { facetas } \\
\text { laminadas, } \\
\text { Infraestruturas } \\
\text { de coroas totais. } \\
\text { Prótese fixa de } \\
\text { até } 3 \text { elementos } \\
\text { anterior ou } \\
\text { posterior até pré- } \\
\text { molares, lentes } \\
\text { de contato, } \\
\text { prótese adesiva } \\
\text { anterior. } \\
\end{array}$ & $\begin{array}{l}\text { Matriz vítrea } \\
\text { com reforço de } \\
\text { partículas de } \\
\text { dissilicato de } \\
\text { lítio }\end{array}$ & $\begin{array}{c}\text { Ácido } \\
\text { sensíveis }\end{array}$ & $300-400$ \\
\hline $\begin{array}{l}\text { Alumina Spinell } \\
\text { (óxidos de } \\
\text { alumínio e } \\
\text { magnésio) } \\
\text { Zircônia } \\
\text { (alumina e óxido } \\
\text { de zircônia) }\end{array}$ & $\begin{array}{l}\text { In Ceram Alumina } \\
\text { (VITA) In Ceram } \\
\text { Spinell (VITA) In } \\
\text { Ceram Zirconia } \\
\text { (VITA) }\end{array}$ & $\begin{array}{c}\text { Coroa total } \\
\text { anterior e } \\
\text { prótese fixa } \\
\text { anterior de até } 3 \\
\text { elementos } \\
\text { Prótese fixa } \\
\text { anterior de } 3 \\
\text { elementos, coroa } \\
\text { posterior, ponte } \\
\text { fixa posterior } 3 \\
\text { elementos até } 1 \\
\text { molar } \\
\end{array}$ & $\begin{array}{l}\text { Fase cristalina } \\
\text { com vidro } \\
\text { infiltrado }\end{array}$ & $\begin{array}{l}\text { Ácido } \\
\text { resistentes }\end{array}$ & $550-650$ \\
\hline Policristalina & $\begin{array}{c}\text { Alumina Pura Lava } \\
\text { (3M); Procera } \\
\text { AllZirkon (Nobel); } \\
\text { Cercon (Degudent); Y- } \\
\text { TZP (VITA); IPS e- } \\
\text { max Zir CAD (Ivoclar) } \\
\text { Zirkonzahn } \\
\text { (Zirkonzahn) }\end{array}$ & $\begin{array}{c}\text { Infraestruturas } \\
\text { de coroas e } \\
\text { próteses fixas de } \\
3 \text { elementos em } \\
\text { dentes anteriores } \\
\text { e posteriores }\end{array}$ & $\begin{array}{l}\text { Estrutura } \\
\text { cristalina, com } \\
\text { melhores } \\
\text { propriedades } \\
\text { mecânicas e } \\
\text { opacidade }\end{array}$ & $\begin{array}{c}\text { Ácido } \\
\text { resistentes }\end{array}$ & $900-1200$ \\
\hline
\end{tabular}

\section{Classificação quanto ao conteúdo (composição)}

A quantidade de cristais na matriz vítrea influencia diretamente na translucidez da cerâmica. As cerâmicas com uma maior qualidade estética têm em sua composição um 
elevado teor de vidro, razão pela qual, são denominadas vítreas, pois imitam melhor as propriedades ópticas do esmalte e da dentina (17).

A porção vítrea das cerâmicas é caracterizada por cadeias de tetraedros de ( $\mathrm{SiO} 4)$ em que cátions de silício estão posicionados no centro de cada tetraedro com ânions oxigênio em cada um dos cantos, resultando tanto em ligações covalentes quanto em iônicas. A composição usual da cerâmica se dá por: $63 \%$ de sílica, $17 \%$ de alumina, $7 \%$ de óxido de boro, $7 \%$ de potássio, $4 \%$ de sódio, $2 \%$ outros óxidos (3).

Cerâmicas vítreas podem ser condicionadas com ácido (ácidos sensíveis) e silanizadas. Apresentam um superior comportamento adesivo, e tem como vantagem o menor desgaste dentário (2). O uso do silano após o condicionamento destas cerâmicas é um método que proporciona a união química entre a fase inorgânica da cerâmica e a fase orgânica do material resinoso, melhorando a capacidade adesiva (21).

Já as cerâmicas cristalinas (ácidos resistentes), como as cerâmicas à base de alumínio, magnésio e zircônia não são passíveis de receber condicionamento ácido (desprovidos de sílica em sua composição). Elas necessitam de jateamento com óxido de alumínio, criando rugosidade na superfície da cerâmica, aumentando a área de contato com o cimento resinoso, sendo contraindicadas para tratamento com facetas laminadas, em que o sucesso depende exclusivamente da adesão $(2,15,22)$. Para esta revisão apenas aspectos relacionados as cerâmicas utilizadas para confecção de facetas laminadas serão abordados.

\section{Classificação quanto ao tipo}

\section{Cerâmica Feldspática}

A primeira cerâmica desenvolvida para uso odontológico foi a cerâmica feldspática ${ }^{9}$. Esta cerâmica, também conhecida como convencional é composta por uma estrutura vítrea, que apresenta uma mistura de feldspato de potássio (K2O Al2O3 6SiO2) ou feldspato de sódio ( $\mathrm{Na} 2 \mathrm{O} \mathrm{Al} 2 \mathrm{O} 36 \mathrm{SiO} 2)$ e uma ou mais fases cristalinas contendo o quartzo ( $\mathrm{SiO} 2$ ) em uma proporção muito inferior ao caulim (23). Quanto maior a resistência do material, menor a sua espessura, sendo a feldspática considerada menos resistente (2).

São vantagens das cerâmicas feldspáticas convencionais: superior estética, estabilidade química, coeficiente de expansão térmica próxima à do dente, menor condutividade térmica, baixa temperatura de fusão, alta resistência à compressão (350 a $450 \mathrm{Mpa}$ ), compatibilidade biológica, além da resistência ao desgaste e aos fluidos orais. Como desvantagens citam: a maior dureza em relação ao esmalte dos dentes, material mais friável, de perfil frágil, técnica laboratorial convencional e demorada, baixa resistência à tração: 20 a $60 \mathrm{Mpa}(9,18,24)$.

Caracterizada como ácido sensível, para as cerâmicas feldspáticas o condicionamento de superfície com ácido fluorídrico no tempo de 60 segundos é uma técnica eficaz na promoção de união mecânica ao agente cimentante resinoso. O condicionamento da superfície interna destas cerâmicas vítreas promove a criação de micro porosidades e promoção de limpeza da peça, aumentando a sua aderência ao substrato dentário $(15,25)$. As cerâmicas feldspáticas convencionais podem ser utilizadas com excelentes resultados para facetas laminadas, porém, as cerâmicas reforçadas apresentam melhores propriedades mecânicas e a possibilidade de estratificação para correções de estética, mesmo após a primeira prova na boca (26) 


\section{Cerâmica reforçada por leucita}

Com o desenvolvimento de cerâmicas prensadas reforçadas por leucita e o avanço na cimentação adesiva, tornou-se viável obter peças cada vez menos espessas e com melhor resistência biomecânica (27).

As cerâmicas vítreas reforçadas por leucita representam um avanço na formulação dos materiais dentários. Comparada a porcelana feldspática convencional, tais cerâmicas apresentam maior peso de leucita incorporado à base de vidro das cerâmicas (em torno de $55 \%$ ), tendo por resultado um aumento relevante da resistência física do conjunto (24).

Esta cerâmica foi disponibilizada sobre o nome comercial IPS Empress Esthetic, integrando o sistema Empress da Ivoclar Vivadent (28). Com cristais de leucita menores e uma distribuição mais homogênea e compacta, alcançou propriedades mecânicas melhores e maiores níveis de translucidez (17).

As propriedades do sistema que utiliza vidros reforçados por leucita são: maior resistência física, resistência flexural: 97 a 180 Mpa, resistência à abrasão semelhante ao esmalte, tempo de processamento laboratorial diminuído, lingotes de cerâmica disponíveis em várias tonalidades, menor formação de poros e contrações (diminuindo as chances de fratura das peças) e translucidez (9).

Também, são indicadas para os seguintes casos: facetas laminadas ou fragmentos em dentes anteriores, recobrimento de próteses unitárias ou múltiplas, inlay, onlay e overlay e coroa unitária. Representando assim, um importante ganho estético $(9,18,24)$. Entretanto, assim como as cerâmicas feldspáticas convencionais, as reforçadas por leucita também são consideradas ácido-sensíveis, com tempo de condicionamento de 60 segundos pelo ácido hidrofluorídrico $(17,22)$.

Vale ressaltar que, a combinação da adesão entre esmalte, dentina e cerâmica, já é bem estabelecida no sistema Empress. Esta união adesiva produz restaurações com excelente integridade mecânica, além de eliminar possíveis defeitos da superfície interna, reduzindo o potencial de fratura (29). No entanto, estas cerâmicas entraram em desuso, principalmente pela razão do elevado coeficiente de expansão térmica e disponibilidade de outras cerâmicas $(9,18,24)$.

\section{Cerâmicas reforçadas por dissilicato de lítio}

Com o surgimento de novas cerâmicas, novos métodos de processamento surgiram e materiais como o dissilicato de lítio, previamente desenvolvido por meio de um tratamento diferenciado durante a queima e da tecnologia CAD/CAM dobrou a resistência flexural mantendo as propriedades estéticas (30). Este sistema permite utilizar na composição $60 \%$ em volume de cristais de dissilicato de lítio, o que faz com que melhore as suas propriedades mecânicas, sem alterar as qualidades ópticas (24).

As propriedades das cerâmicas reforçadas por dissilicato de lítio são: maior disponibilidade de cores, aperfeiçoamento no processo de confecção laboratorial, resistência flexural triplicada (300 a $400 \mathrm{MPa}$ ), excelente estética, melhor capacidade adesiva, translucidez, opacidade e fluorescência semelhante ao dente, resistência à abrasão semelhante ao esmalte, menor formação de poros e contrações, diminuindo as chances de fratura das peças (31).

Substituindo as reforçadas por leucita, devido às melhores propriedades estéticas e maior resistência $(9,31)$, as cerâmicas à base de dissilicato de lítio são indicadas para facetas laminadas ou fragmentos em dentes anteriores, dentre outras $(9,18,24)$. 
Além disso, tornaram-se mais empregadas, pois os cristais presentes em sua composição possuem índice de refração semelhante ao da matriz vítrea, permitindo o aumento do volume sem que haja perda da translucidez (27). O acréscimo destes cristais na formulação, dispersas em uma matriz vítrea de forma interlaçada, dificulta a propagação de trincas em seu interior e favorece as propriedades mecânicas sem, contudo, comprometer as propriedades ópticas destas cerâmicas vítreas (32).

Consideradas também como ácido-sensíveis, as cerâmicas de dissilicato de lítio, apresentam um tempo de condicionamento reduzido em comparação às demais cerâmicas, 20s pelo ácido hidrofluorídrico (33), o que otimiza o tempo clínico de consultório. A exemplo dessas cerâmicas têm-se: IPS Empress 2 (Ivoclar), IPS e.max (Press ou CAD) para as cerâmicas injetadas ou fresadas, e o IPS e.max ceram, que permite confeccionar coroas extratificadas sobre copping de e.max Press (34).

O sistema cerâmico (IPS E-max, Ivoclar Vivadent, Schaan, Liechtenstein) tem -se destacado no mercado por ser uma cerâmica à base de dissilicato de lítio de alta resistência resultando em boa resolução estética. Ainda, não é citotóxico, durabilidade do tratamento independente da técnica utilizada, níveis de desgaste in vivo das cerâmicas semelhantes à média de desgaste do esmalte natural e com opções de cimentação convencional, adesiva ou autoadesiva $(31,35,36)$.

Suas principais indicações são a confecção de coroas unitárias em cerâmica pura, inlays, onlays, facetas laminadas e confecção de próteses parciais fixas de três elementos, envolvendo dentes anteriores e posteriores (35-37). Restaurações de cerâmica pura com evidência clínica de longo prazo com resultados estáveis, precisos e realistas são citados como vantagens desse sistema (36).

Dessa forma, devido a constante busca do profissional pela máxima preservação das estruturas sadias, novas técnicas e o desenvolvimento de materiais oferecem a possibilidade de realizar tratamentos restauradores que recuperem o tecido dental perdido. Devolvendo, assim, as características naturais, respeitando os princípios conservadores, condições funcionais e biológicas envolvidas (38). As facetas laminadas à base de dissilicato de lítio são peças capazes de solucionar grandes problemas estéticos aliando beleza e resistência $(32,38,39)$.

Tendo em vista que o sucesso do tratamento está diretamente relacionado à adequada escolha do material cerâmico, selecioná-la baseada apenas na sua transluscência pode levar a uma indicação errônea (16). Portanto, o sistema cerâmico reforçado por dissilicato de lítio IPS Emax (Ivoclar Vivadent) possui excelentes propriedades ópticas, independente da tonalidade do dente natural, aliado à uma elevada resistência mecânica, sendo frequentemente indicado para confecção de facetas laminadas $(36,40)$.

\section{Conclusão}

Cerâmicas feldspáticas convencionais, leucíticas e dissilicato de lítio podem ser usadas para facetas laminadas, devido às propriedades ópticas, biomecânicas e o alto teor de sílica na composição desses laminados. Entretanto, as cerâmicas à base de dissilicato de lítio são as mais indicadas pois dispõem de maior resistência flexural, excelente estética e melhor capacidade adesiva em comparação às outras cerâmicas. O sistema e.max se diferencia no mercado em virtude de sua versatilidade e proporciona excelente resultado estético sem comprometer o desempenho mecânico das restaurações.

\section{Conflito de Interesses}

Os autores declaram que não existe conflito de interesse. 


\section{Referências}

1. Rekow ED, Silva NRFA, Coelho PG, Zang Y, Guess P, Thompson VP. Performance of dental ceramics: challenges for improvement. J Dent Res 2011; 90(8): 937-952.

2. Amoroso PA, Ferreira BM, Torcato BL. Cerâmicas odontológicas: propriedades, indicações e considerações clínicas. Rev Odontol Araçatuba. 2012; 33(2):19-25.

3. Anusavice KJ, Shen C, Rawls H.R. - Phillips Materiais Dentários. 12. ed. Rio de Janeiro: Elsevier, 2013.

4. Okida RC, Vieira WSC, Rahal V, Okida DDS. Lentes de Conato: restaurações minimamente invasivas na solução de problemas estéticos. Rev Odontol Araçatuba 2016; 37(1): 53-59.

5. Aquino APT, Cardoso PC, Rodrigues MB, Takano A.E, Porfírio W. Facetas de porcelana: solução estética e funcional. Clínica Int J Braz Dent 2009; 5(2): 142-152.

6. Cardoso C, Cardoso LC, Decurcio RA, Junior LM. Restabelecimento Estético Funcional com Laminados Cerâmicos. ROBRAC 2011; 20(52): 88-93.

7. Mclaren EA, Figueira J. Updating Classifications of Ceramic Dental Materials: A Guide to Material Selection, Compendium 2015; 36(6):400-406.

8. Alves GS, Miranda ME, Santos VMA, Guimaraes PS, Sinhoretti M.A. Avaliação da rugosidade superficial e da dureza de diferentes cerâmicas odontológicas. RGO 2005; 53(3): 220-225.

9. Conceição EN. Laminados cerâmicos. In. Dentística: saúde e estética. 2 eds. Porto Alegre: Artmed, 2007; p.478-501.

10. Pires LA, Novais PMR, Araújo VD, Pergoraro LF. Effects of the type and thickness of ceramic, substrate, and cement on the optical color of a lithium disilicate ceramic. J Prost Dent 2017; 117: 144-149.

11. Reis GR, Vilela ALA, Silva FP, Borges MG, Santos Filho PC, Menezes MS. Minimally Invasive Aprocch In Esthetic Dentistry: Composite Resin Versus Ceramics Veneers. Biosci. J 2017; 33(1): 238-246.

12. Soares PV, Zeola LF, Souza PG, Pereira FA, Milito GA, Machado AC. Reabilitação Estética do Sorriso com Facetas Cerâmicas Reforçadas por Dissilicato de Lítio. ROBRAC 2012; 21(58): 538-543.

13. Silva WO, Almeida RF, Leal L, Carvalho Junior EU. Recontorno estético multidisciplinar com cirurgia plástica gengival e laminados cerâmicos de dissilicato de lítio: passo a passo. R Dental Press Estét 2015; 12(4):101-118.

14. Magne P. Uso de Encerramento por Acréscimo e Ensaio Direto Intraoral para Preservação de Esmalte com Facetas Laminadas de Porcelana. Clínica: Inter J Braz Dent 2007; 3(1): 25-31.

15. Gonzalez MR, Ritto FP, Lacerda RAS, Sampaio HR, Monnerat AF, Pinto BD. Falhas em Restaurações com facetas laminadas: uma revisão de literatura de 20 anos. RBO 2012; 69(1): 43-48.

16. Neto JMA, Furtado KRS, Baumberger MCA, Duarte IKF, Trujillo, Alves EVR, Medeiros MLBB et al. Cerâmicas odontológicas: Uma Revisão de Literatura. Revista Eletrônica Acervo Saúde 2020; 40: 1-10.

17. Kina, S. Cerâmicas dentárias. Rev. Dental Press Estét. 2005; 2(2):111- 128.

18. Gomes EA. Assunção WG, Rocha, E. P.; Santos, P. H. Cerâmicas odontológicas: o estado atual. Cerâmica [online] 2008; 54(331): 319-325.

19. Helvey GA. Classifying Dental Ceramics: Numerous Materials and Formulations Available for Indirect Restorations, Compendium 2014; 38-43.

20. Raposo LH. Restaurações totalmente cerâmicas, características, aplicações clínicas e longevidade. Pro-odonto prótese e dentística 2014; 2:1-66.

21. Miyashita E, Fonseca AS. Odontologia estética: o estado da arte. São Paulo: Artes Médicas, 2004.

22. Soares PV, Spini PH, Carvalho VF, Souza PG, Gonzaga R C, Tolentino AB. Esthetic rehabilitation with laminated ceramic veneers reinforced by lithium disilicate. Quintessenc e Int 2014; 45(2): 129-133.

23. Bottino MA, Snellaert A, Bergoli CD, Ozcan MC, Valandro LF. Effect of ceramic etching protocols on resin bond strength to a feldspar ceramic. Oper Dent 2015; 40(2): 40-46. 
24. Baratieri LN, Cardoso PC, Decurcio RA, Machado RG. Restaurações Cerâmicas Parciais-Facetas. In: BARATIEIRI, L.N., et. al, Odontologia Restauradora: Fundamentos e possibilidades. São Paulo: 2 Ed. Santos, 2015. cap. 15, pag. 593-638.

25. Fuzo A Bottino MA, Faria R. Facetas laminadas: funcionais, estéticas e preservadoras. Implant News 2014; 10(4): 34-40.

26. Buso L, Ferreira JV. Facetas laminadas sistema empress esthetic. Clínica Inter J Bras Dent. 2006; 2(3): 306-314.

27. Guess PC, Schultheis S, Bonfante EA, Coelho PG, Ferencz JL, Silva NR. All ceramic systems: laboratory and clinical performance. Dent Clinic North Am 2011; 3(55): 333-352.

28. Ivoclar Vivadent [internet]. IPS Empress Esthetic;2006. [citado em 2020 jan30]. Disponívelem:http://Ivoclar+Vivadent.+IPS+empress+esthetic\&oq=Ivoclar+Vivadent.+IPS+empress +esthetic\&gs_l=psyab.

29. Kina, S. Protocolo clínico para utilização de uma nova cerâmica vítrea reforçada por leucita. Rev Dent Press Estet 2005b; 2(4): 23-67.

30. Fasbinder DJ, Dennison JB, Heys D, Neiva GA. Clinical evaluation of chairside lithium disilicate CAD/CAM crowns: a two-year report. J Am Dent Assoc 2010; 141(2): 10-14.

31. Wendling M, Santos L, Donassollo TA, Donassollo SH. Reabilitação Funcional e Estética em Dentes Anteriores e Posteriores - Relato de Caso. Clínica Inter J Braz Dent 2016; 12(2): 172-179.

32. Aguiar EMG, Rodrigues R B, Lopes CCA, Júnior CDS, Soares C J, Novais VR. Diferentes sistemas cerâmicos na reabilitação oral: relato de caso clínico. ROBRAC 2016; 25(72): 31-36.

33. Rodrigues RB, Verissimo C, Pereira RD, Queiroz CL, Novais VR, Soares CJ. Clareamento dentário associado a facetas Indiretas em cerâmica: Abordagem minimamente invasiva. ROBRAC 2012; 21(59): 520-525.

34. Ivoclar Vivadent [internet]. IPS e.max dissilicato de lítio; 2009 [citado em 2020 jan 30]. Disponível em: http://ivoclarvivadent.com.br.

35. Neves GGA, Maia EAV, Dobranszki NPAC. Facetas laminadas com mínimo desgaste - protocolo de interesse clínico. ROBRAC 2014; 4(1): 23-31.

36. Ivoclar Vivadent [internet]. IPS e.max; 2018 [citado em 2020 jan 30]. Disponível em: http://ivoclarvivadent.com.br.

37. Mazioli CG, Peçanha MM, Daroz LG, Siqueira CA, Fraga MAA. Resistência de união de diferentes cimentos resinosos a cerâmica à base de dissilicato de lítio. Rev Odontol Unesp 2017; 46(3): 174-178.

38. Shibayama R, Tiossi R, Queiroz M, Dallazen E, Campaner M. Reabilitação estética dos elementos anteriores utilizando o sistema IPS e max. Rev Odontol Araçatuba 2016; 37(2): 09-16.

39. Vaz MV, Edenize CV, Celha BC, Juliana C, Marcos A, João B. Utilização do ensaio restaurador como guia de desgaste em reabilitação estética com sistema IPS e.max: caso clínico. ROBRAC 2015; 24(68): 6-10.

40. Steffens SP, Muraro D, Donassollo TA; Donassollo SH. Inter-Relação Dentística x Prótese x Periodontia para Reabilitação Estética e Funcional: Relato de Caso. Clínica Inter J Braz Dent 2016; 12(2): 156-162.

41. Vieira AC, Oliveira MCS, Andrade AC, Sampaio, NM, Nascimento LB, Jaqueline JA. Reabilitação estética e funcional do sorriso com restaurações cerâmicas de diferentes espessuras. Rev. Odontol. Araçatuba 2018; 39(3): 32-38. 